\title{
Clonal diversity and antimicrobial resistance of Staphylocaccus pseudintermedius isolated from canine pyoderma
}

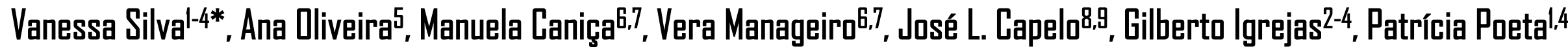 \\ IMicrobiology and Antibiotic Resistance Team (MicroART), Department of Veterinary Sciences, University of Trás-os-Montes and Alto Douro (UTAD), Vila Real, Portugal; \\ 2Department of Genetics and Biotechnology, University of Trás-as-Montes and Alta Douro, Vila Real, Partugal; \\ ${ }_{3}^{3}$ Functional Genomics and Proteomics Unit, University of Trás-os-Montes and Alta Douro (UTAD), Vila Real, Partugal; \\ ${ }^{4}$ Assaciated Laboratory for Green Chemistry (LADV-REDUIMTE), University NDVA of Lisboa, Lisboa, Caparica, Partugal; \\ ${ }_{5}^{5}$ Faculty of Veterinary Medicine, University Lusófona de Humanidades e Tecnologias, 1749-024 Lisboa, Portugal; \\ ENational Reference Laboratory of Antibiotic Resistances and Healthcare Assaciated Infections (NRL-AMR/HAl), Department of Infectious Diseases, National Institute of Health Dr Ricarda Jarge, Av. Padre Cruz, I649-DI6, Lisbon, Portugal; \\ Tentre for the Studies of Animal Science, Institute of Agrarian and Agri-Food Sciences and Technologies, Oporta University, Oporta, Portugal; \\ ${ }^{8}$ BIDSCDPE Group, LADV回REDUIMTE, Chemistry Department, Faculty of Science and Technology, NDVA University of Lisbon, Almada, Portugal;

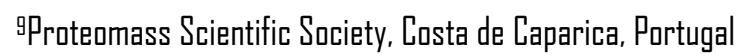 \\ *vanessasilva回utad.pt
}

\section{Introduction "\%:}

Staphylacaccus pseudintermedius is a predominant cause of skin infections in dogs and the most common causative agent of pyoderma. Methicillin-resistant $S$. pseudintermedius (MRSP) have been identified in increasing frequencies in canine pyoderma. MRSP strains are usually resistant to several classes of antibiatics which leads to therapeutic failure and, potentially, zoonotic problems. This study aimed to characterize the antimicrobial resistance and genetic lineages of $S$. pseudintermedius isalated from canine pyoderma.

\section{Methods $\%$}

Sixty-one $S$. pseudintermedius were isolated from dogs with pyoderma in a veterinary hospital. The presence of mecA gene was detected by PCR. Antimicrobial susceptibility testing was performed by the Kirby-Bauer disk diffusion methad against 17 antimicrobial agents. Multilocus-sequence-typing (MLST) was perfarmed in all MRSP isolates as previously described (https://pubmlst.org/).

\section{Results \%:}

From the 61 isolates, 31 harbored the mecA gene and were therefore classified as MRSP. The majority of $S$. pseudintermedius isolates showed resistance to penicillin, erythramycin, clindamycin, tetracycline and trimethoprim-sulfamethaxazole (Figure I). Mast MRSP also shawed resistance to aminaglycasides. MSRP isolates were ascribed to 9 previously described sequence types (ST): STIZ3, ST727, ST339, ST537, ST45, STI0Z9, STIIZ, ST1468, ST71; and to 5 ST described far the first time in this study: ST2024, ST2025, ST2026, ST2027 and ST2028 (Figure 2).

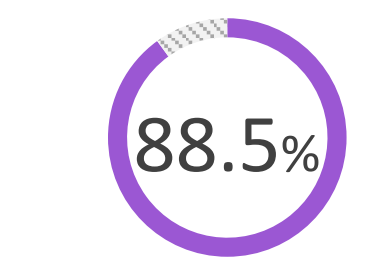

Penicillin

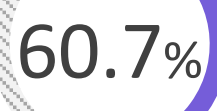

Erythromycin and alindamycin

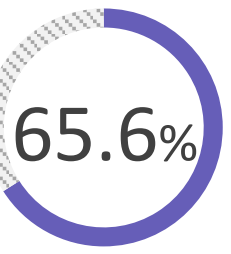

Tetracycline

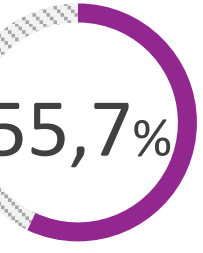

Trimethoprimsulfamethoxazole

Figure I. Percentages of the mast prevalent resistances found amang the $S$. pseudintermedius.

\section{8\%: Canclusions}

These results show that more than half of $S$. pseudintermedius isolated from pyoderma were resistant to methicillin. There was a difference in the antimicrabial susceptibility pattern between methicillin-resistant and -sensible $S$. pseudintermedius, in particular, for aminaglycasides. Furthermore, there was a high diversity of genetic lineages among MSRP causing pyoderma.

\section{Acknowledgements:}

This work was also supported by the Assaciate Laboratory for Green Chemistry-LADV which is financed by national funds from FCT/MCTES (UID/DU//50006/2020). Vanessa Silva is grateful to FCT (Fundação para a Ciência e a Tecnologia) for financial support through PhD grant SFRH/BD/137947/2018.

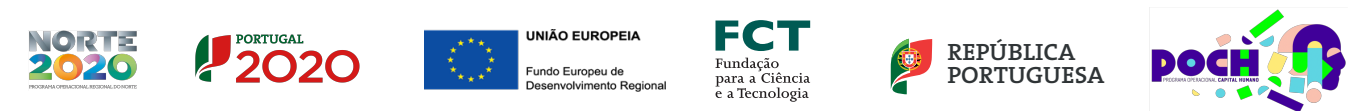

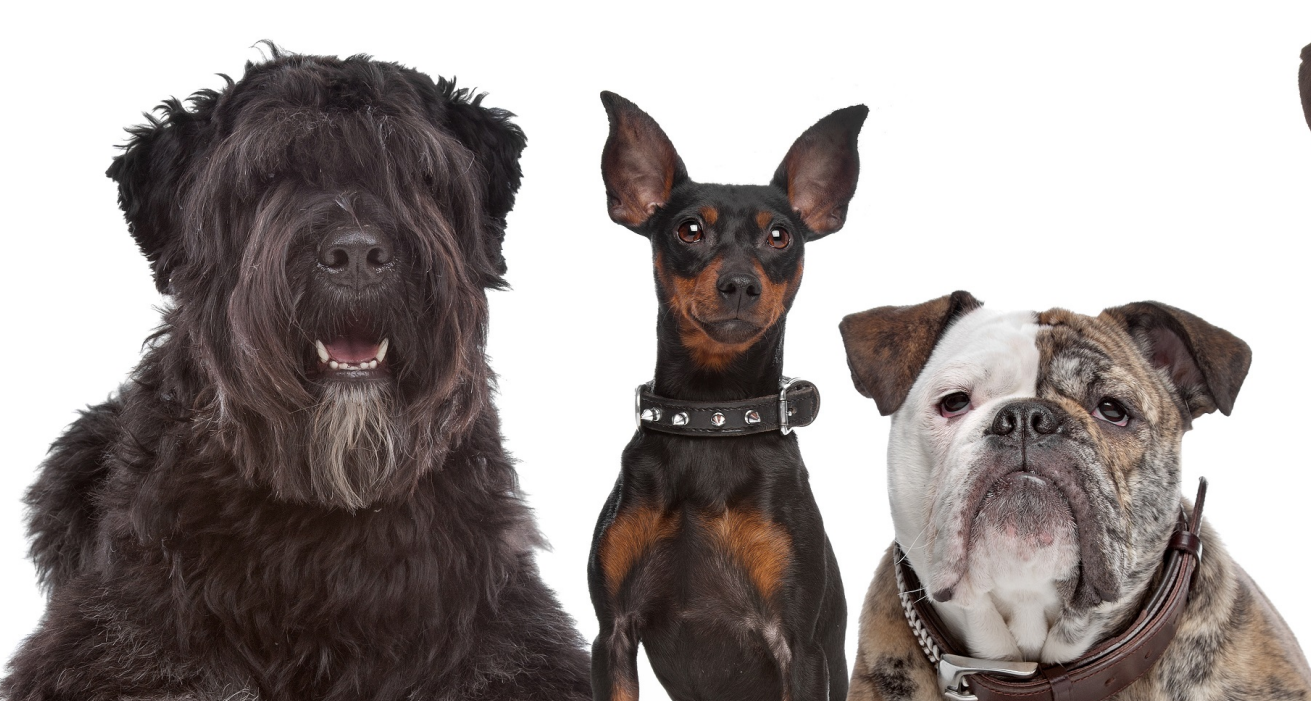
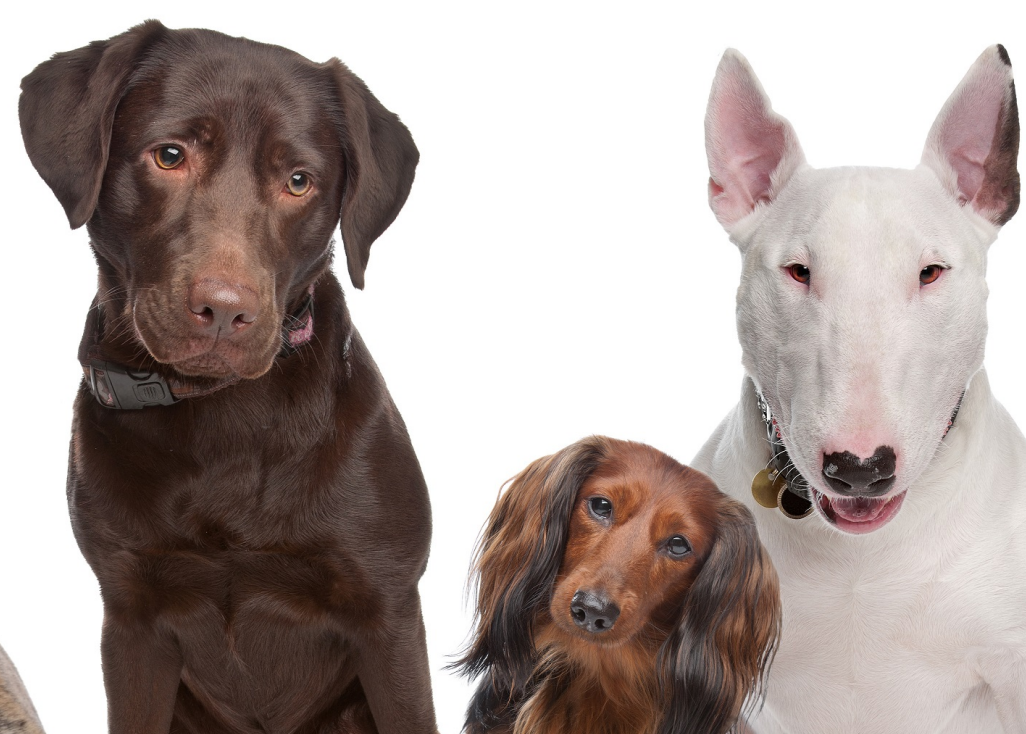

Figure 2. Distribution of 15 different STs among MRSP.

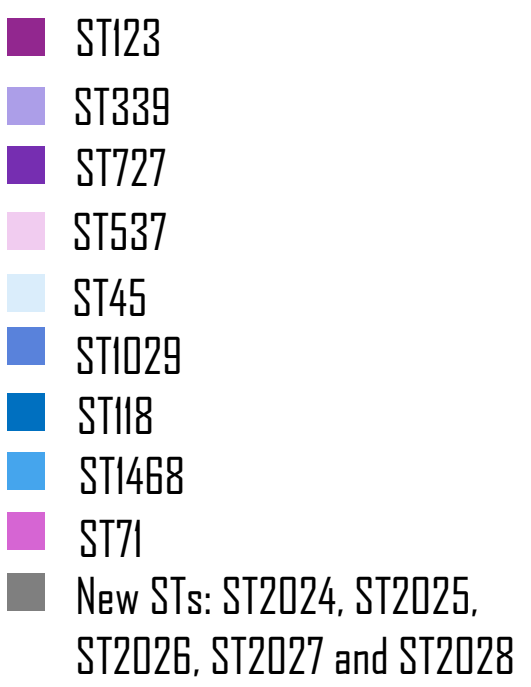

ST20Z6, ST2027 and ST2028 